Revista Universo Contábil, ISSN 1809-3337

FURB, v. 6, n.1, p. 67-84, jan./mar., 2010

doi:10.4270/ruc.2010104

Disponível em www.furb.br/universocontabil

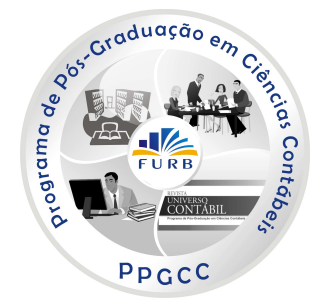

\title{
ANÁLISE DA APLICABILIDADE DO TIME-DRIVEN ACTIVITY-BASED COSTING EM EMPRESAS DE PRODUÇÃO POR ENCOMENDA*
}

\section{ANALYSIS OF THE APPLICABILITY OF THE TIME-DRIVEN ACTIVITY-BASED COSTING IN MAKE-TO-ORDER COMPANIES}

Antônio Artur de Souza

$\mathrm{PhD}$ in Management Sciences pela University of Lancaster - UK Professor do Mestrado em Ciências Contábeis da UFMG Endereço: Avenida Antônio Carlos, nº 6627 - Bairro Pampulha CEP: 31270-901 - Belo Horizonte/MG - Brasil E-mail : artur@face.ufmg.br Telefone: (31) 3409-7057

Ewerton Alex Avelar

Graduação em Ciências Contábeis pela UFMG Endereço: Avenida Antônio Carlos, nº 6627 - Campus Pampulha. CEP: 31270-901 - Belo Horizonte/MG - Brasil E-mail: ewerton@face.ufmg.br Telefone: (31) 3409-7057

Terence Machado Boina Graduação em Ciências Contábeis pela UFMG Endereço: Avenida Antônio Carlos, nº 6627 - Campus Pampulha. CEP: 31270-901 - Belo Horizonte/MG - Brasil E-mail: terencemb@face.ufmg.br Telefone: (31) 3409-7057

Simone Leticia Raimundini Doutoranda do Programa de Pós-Graduação em Administração da UFRGS Professora da Departamento de Ciências Contábeis e Atuariais da Universidade Federal do Rio Grande do Sul - UFRGS Endereço: Av. João Pessoa, 52 - sala 23 CEP: 90040-000 - Porto Alegre/RS - Brasil

E-mail: simone.raimundini@ufrgs.br Telefone (51) 3308-3323

Artigo recebido em 19.11.2008. Revisado por pares em 13.02.2009. Reformulado em 25.03.2009. Recomendado em 26.03.2009 por Ilse Maria Beuren (Editora). Publicado em 02.02.2010. Organização responsável pelo periódico: FURB. 


\section{RESUMO}

Este trabalho apresenta os resultados de uma pesquisa que objetivou analisar a percepção dos gestores de atividades selecionadas sobre a aplicabilidade da abordagem Time-driven do custeio baseado em atividades (Time-driven Activity-Based Costing - TDABC) em uma empresa de produção por encomenda (EPE) localizada na região metropolitana de Belo Horizonte/MG. A pesquisa, exploratória e qualitativa, adotou como metodologia o estudo de caso. Diversas técnicas de coleta de dados foram utilizadas, tais como entrevistas semiestruturadas, análise documental e observação participante. Os resultados da pesquisa demonstram que o TDABC aprimora o uso dos direcionadores de custos e exige que as atividades identificadas no $\mathrm{ABC}$ tradicional sejam segregadas em sub-atividades, para que seja possível mensurar o tempo gasto por meio das equações de tempo (time equations). Verificou-se que a aplicação do TDABC em um ambiente de produção por encomenda é bastante complexa. A imprevisibilidade inerente às EPEs dificulta a elaboração das time equations, fundamentais para a implantação do TDABC, normalmente, limitando seu uso por parte desse tipo de empresa. Destacam-se algumas limitações da pesquisa: os resultados não podem ser generalizados indiscriminadamente, uma vez que se tratou de um estudo de caso único e o setor de atuação da EPE estudada é bastante peculiar. Ressalta-se, ainda, a escassez de pesquisas sobre o TDABC, principalmente, no Brasil.

Palavras-chave: Empresas de produção por encomenda. Custeio Baseado em Atividades. Abordagem Time-driven.

\section{ABSTRACT}

This paper presents the results of a research whose objective was to analyze the managers' perception of the applicability of the time-driven activity-based costing approach (TDABC) in a make-to-order company (MTO) located in the metropolitan area of Belo Horizonte (MG). The methodology applied in this exploratory qualitative research was the case study. Several data collection techniques were used, such as semi-structured interviews, documental analysis and participant observation. The research results show that the TDABC improves the use of cost drivers and forces the activities identified in the $A B C$ to be grouped in subactivities so one can measure the time spent by means of time equations. Applying the TDABC in a make-to-order environment proved to be rather complex. The inherent unpredictability of an MTO company makes it difficult to create time equations, which are fundamental to implement the TDABC, thus restricting its use in that kind of company. Some restrictions stand out: the results can not be indiscriminately generalized, once they dealt with a single case study, and the sector covered by the MTO company surveyed is rather peculiar. It is also important to point out the lack of researches on TDABC, especially in Brazil.

Keywords: Make-to-order companies. Activity-Based Costing. Time-driven approach.

\section{INTRODUÇÃO}

O crescimento da competitividade empresarial tem sido um dos fatores determinantes na gestão das empresas nas últimas décadas. Os níveis de dinamismo e incerteza do mercado, que se elevam de forma contínua, refletem uma hostilidade ambiental crescente, que ameaça a sobrevivência de muitas empresas (BASTOS, 2003). Brandão e Guimarães (1999) afirmam 
que tal contexto traz como conseqüências o desenvolvimento e a incorporação de novas tecnologias e modelos de gestão. Assim, as empresas, de modo geral, precisam desenvolver e utilizar ferramentas de gestão que lhes capacitem competir de forma sustentável.

Nesse sentido, Kaplan e Cooper (1998) destacam que, no atual ambiente em que as empresas atuam, a gestão de custos se torna um dos aspectos de extrema importância para a sustentação da competitividade. Entretanto, para que as decisões relacionadas a custos sejam adequadas, de maneira geral, suas informações devem representar ao máximo a realidade na qual se encontra a empresa, sendo que a qualidade das informações de custos está estreitamente relacionada ao método de custeio utilizado para gerá-las (ATKINSON et al., 2008). Dentre os principais métodos existentes na literatura, o custeio baseado em atividades (Activity Based Costing $-A B C$ ), de uma forma geral, é indicado por diversos autores, tais como Brimson (1996), Kaplan e Cooper (1998), Boisvert (1999), Cokins (1999), Atkinson et al. (2008), Hirsh (2000), e Cokins e Hicks (2007), como superior aos demais, em termos de capacidade de gerar informações úteis para a tomada de decisão gerencial.

Contudo, o ABC é alvo de uma série de críticas relacionadas à sua eficácia na geração de informações úteis para subsidiar o processo decisório dos gestores. Muitas destas críticas são fundamentadas na teoria das restrições proposta por Goldratt e Cox (1997). Estes autores questionam a validade das informações de custos - e, conseqüentemente, das provenientes do ABC - no processo decisório empresarial. Autores como Corbett Neto (1997), e Roni e Geri (2005) criticam algumas limitações do ABC, tais como o fato dessa metodologia usualmente desconsiderar os gargalos de produção e considerar que a relação entre o consumo de recursos e o nível de atividades é linear.

No intuito de atenuar algumas limitações do ABC, Kaplan e Anderson (2004) propõem outra abordagem para esta metodologia: o Time-driven $\mathrm{ABC}$ (TDABC). Esta abordagem utiliza novas ferramentas, tais como as equações de tempo (time equations). Além disso, dentre outras inovações, elimina a necessidade de se realizarem entrevistas com funcionários, que serviriam para avaliar em quais atividades eles trabalham durante determinado período. Destaca-se que, segundo Kaplan e Anderson (2007b), esta é uma das principais falhas do $\mathrm{ABC}$, pois tais entrevistas demandam enorme quantidade de tempo.

Este trabalho apresenta os resultados de uma pesquisa que visou analisar a percepção dos gestores, de atividades selecionadas de uma empresa de produção por encomenda (EPE), sobre a aplicabilidade do TDABC. Salienta-se que as empresas que atuam no setor de produção por encomenda, de modo geral, têm a essência de sua competitividade calcada nas informações de custos (CAMPOS, 2003; MEGLIORINI, 2003; SOUZA et al., 2005).

Este artigo está estruturado em sete seções, contando com esta introdução. Nas seções de 2 a 4, é desenvolvida a fundamentação teórica da pesquisa. Na seção 5, descreve-se a metodologia de pesquisa. Os resultados do estudo de caso são apresentados na seção 6 . $\mathrm{Na}$ seção 7, apresentam-se as conclusões do trabalho.

\section{EMPRESAS DE PRODUÇÃO POR ENCOMENDA (EPES)}

São classificadas como EPEs as empresas que produzem uma grande variedade de produtos em um volume relativamente baixo (HENDRY, 1998). Essas empresas, normalmente, elaboram produtos customizados, que seguem as especificações dos clientes (YEH, 2000). Nesse sentido, de acordo com Palomino (2001), os equipamentos de produção das EPEs devem ser flexíveis e de finalidade geral, para atender às múltiplas requisições de seus clientes.

Corrêa e Corrêa (2007) apresentam a seguinte classificação de processos produtivos: a) Processo por tarefa - caracterizado pela produção de pequenos lotes, de uma grande 
variedade de produtos; b) Processo de lotes (batch) - é similar ao processo por tarefa, embora apresente maior especialização dos recursos e algumas economias de escala; c) Processo em linha - caracterizado pela produção de peças discretas (em unidades), que passam de uma estação de trabalho a outra, numa taxa pré-determinada; e, d) Processo contínuo caracterizado pelo processamento de materiais não discretos, em estruturas fabris geralmente automatizadas.

Sob a referida classificação, podem ser consideradas EPEs as empresas que apresentam os processos por tarefa e de lotes. Exemplos dessas empresas, segundo aqueles autores, são: ferramentarias, fábricas de móveis de cozinha por encomenda, fábricas de máquinas convencionais (processo por tarefa); indústrias químicas de especialidades, indústrias de alimentos e estamparias de montadoras de veículos (processo de lotes).

Stevenson et al. (2005), por sua vez, apresentam dois tipos diferentes de EPEs. As que fabricam produtos ao longo de um contrato para determinado cliente (Repeat Business Customiers - RBC) estão inseridas em um ambiente relativamente menos instável, embora a incerteza inerente ao setor de atuação não consiga ser excluída. As que fabricam produtos que dificilmente são repetidos (Versatile Manufacturing Companies - VMC) possuem um mercado mais complexo, no qual são necessárias soluções mais sofisticadas e particulares, de acordo com cada pedido. As empresas classificadas como Make-to-stock (MTS) elaboram produtos padronizados, fundamentalmente para estoques. A Figura 1 apresenta essa classificação. Na pesquisa apresentada neste trabalho, a EPE estudada é classificada como VMC, de acordo com Stevenson et al. (2005). Na classificação de Corrêa e Corrêa (2007), o processo produtivo da referida EPE é por tarefa.

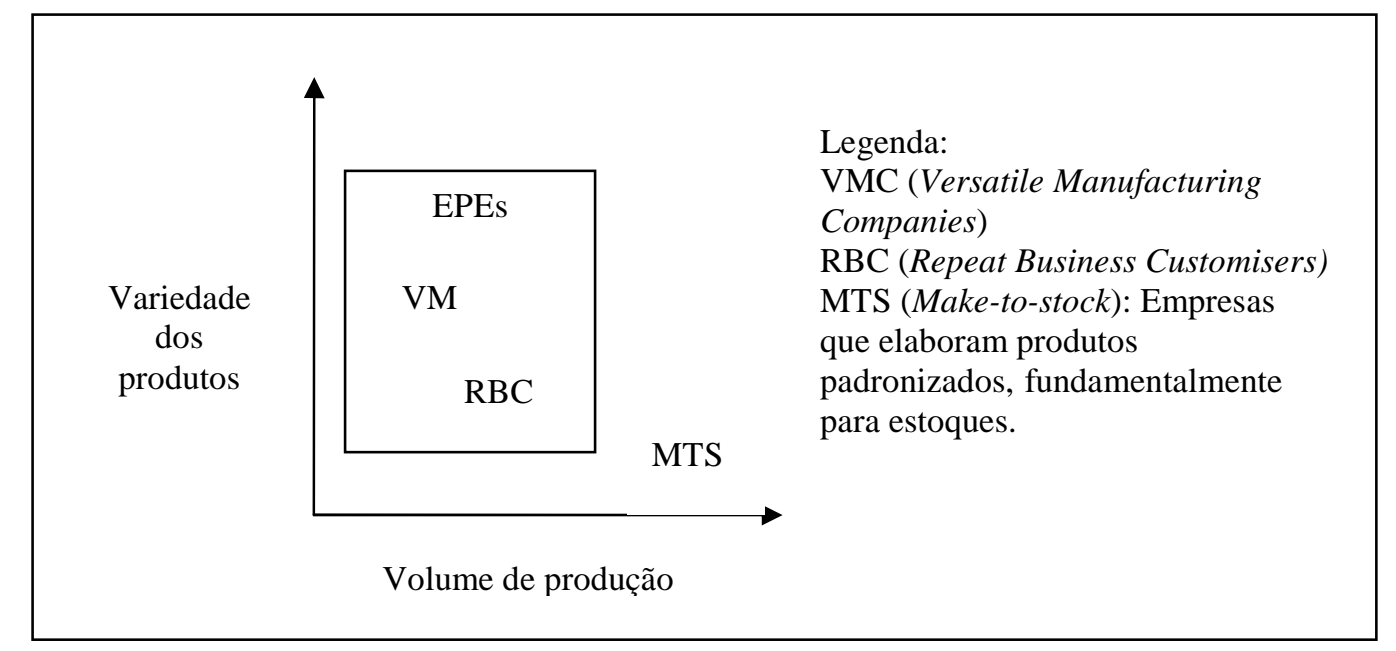

Figura 1 - Classificação de sistemas produtivos baseada em volume e variedade Fonte: adaptado de Stevenson et al. (2005, p. 872).

A complexidade dos sistemas produtivos das EPEs faz com que a tomada de decisão gerencial se torne um processo difícil, uma vez que geralmente está fundamentada em informações de custos. Souza et al. (2005) enumeram uma série de decisões importantes para a competitividade das EPEs, que necessitam dessas informações: terceirização, ampliação da capacidade produtiva e compra de materiais e máquinas. Campos (2003) ressalta a importância das informações de custos para os gestores das EPEs no processo decisório eficaz, principalmente aquelas referentes a preços de produtos. Megliorini (2003) comenta que o processo de formação de preço nas EPEs é fundamental para a competitividade e depende do processo de estimação de custos. Conforme Cokins e Hicks (2007), a qualidade das informações de custos está associada ao método de custeio utilizado para gerá-las. 


\section{CUSTEIO BASEADO EM ATIVIDADES (ABC) E A TOMADA DE DECISÃO}

Existem diversos métodos de custeio citados na literatura e utilizados no ambiente empresarial. Segundo Megliorini (2003), os mais conhecidos e utilizados são: o custeio por absorção, o custeio variável (ou direto) e o ABC. Neste artigo, a discussão se limita ao $\mathrm{ABC}$, por ser o tópico estudado na pesquisa.

Kaplan e Anderson (2007a) relatam que o ABC surgiu na década de 1980 como uma resposta ao custeio tradicional (custeio por absorção), uma vez que esse método não fornecia o suporte necessário aos tomadores de decisão. Para Hansen e Mowen (2006), o ABC é um método de custeio em que os custos inicialmente são atribuídos a atividades e depois aos objetos de custos (produtos, clientes, dentre outros). Nesse sentido, de acordo com Latshaw e Cortese-Danile (2002), o ABC tem duas finalidades: administrar os custos indiretos (gestão baseada em atividades - Activity-based Management) e fornecer informações mais precisas sobre os custos individuais dos produtos e serviços.

Kaplan e Cooper (1998) destacam que o objetivo desse método de custeio é voltado para o longo prazo, nos quais todos os custos podem ser considerados variáveis. Assim, a alocação de custos fixos e indiretos justifica-se sob tal contexto, mesmo com a aparente distorção de algumas informações.

De acordo com Grunow et al.(2005), o ABC utiliza uma série de direcionadores para alocar os custos indiretos de produção aos objetos de custos, o que lhe pode conferir maior precisão. Atkinson et al. (2008) definem que os direcionadores que associam os custos dos recursos às atividades são denominados direcionadores de recursos (ou direcionadores primários) e os direcionadores que associam os custos das atividades aos objetos de custos são denominados de direcionadores de atividades (ou direcionadores secundários).

Segundo Kaplan e Cooper (1998), os direcionadores de atividades podem ser classificados em três diferentes categorias: a) direcionadores de transação - avaliam a frequiência com a qual uma atividade é executada (número de setups realizados, por exemplo); b) direcionadores de duração - representam o tempo necessário para se realizar uma atividade (tempo de setups, por exemplo); e, c) direcionadores de intensidade - indicam os esforços distintos necessários para executar uma atividade (realização de setups em máquinas muito complexas, por exemplo).

Para a adequada operacionalização do ABC, as atividades devem ser classificadas em quatro grupos distintos: unidade, lote, produto e suporte às instalações (KAPLAN e COOPER, 1998; ATKINSON et al., 2008; HIRSH, 2000). As atividades classificadas no nível da unidade se referem às necessárias para elaborar apenas uma unidade de um determinado produto (mão-de-obra direta, por exemplo). Por sua vez, as atividades classificadas no nível do lote são as demandadas cada vez que um lote do produto é fabricado (setups de máquinas, por exemplo). As atividades no nível do produto são as necessárias para a elaboração de determinado produto (projeto, por exemplo). Por fim, as atividades no nível das instalações são as que dão suporte à estrutura geral da empresa (gerência da fábrica, por exemplo).

O ABC pode ser segregado em duas abordagens: Estratégica e Operacional. A primeira abordagem tem o propósito de mensurar os custos de uma forma mais sintética (por grupo e produtos ou unidades de mercado, por exemplo), isto é, agregada para subsidiar a tomada de decisões estratégicas na empresa. Já a segunda abordagem tem como objetivo expressar as informações de custos de forma analítica, notadamente para auxiliar as decisões de âmbito operacional da empresa (KAPLAN; COOPER, 1998). 
No que tange à tomada de decisão, há controvérsias na literatura sobre o $\mathrm{ABC}$. Diversos autores têm ressaltado a importância do $\mathrm{ABC}$ como ferramenta gerencial para os gestores. Cokins e Hicks (2007) afirmam que o ABC melhora as decisões das empresas ao apresentar as relações de causa e efeito das atividades. Hungarato e Sant'Anna (2005) destacam algumas vantagens da utilização do ABC: a) alocação dos gastos indiretos aos objetos de custos, por meio dos direcionadores de custos; b) minimização dos problemas de arbitrariedade dos critérios de rateio dos custos indiretos, que são geralmente utilizados no custeio por absorção; c) a melhoria contínua dos processos da empresa; e, d) a melhor precificação dos produtos e a apuração de rentabilidade por cliente/mercado. Pode-se dizer que o $\mathrm{ABC}$ supera os métodos de custeio tradicionais, uma vez que reconhece que os recursos são resultados de atividades ou transações, focando a atenção dos gerentes nos recursos que eles podem controlar (HIRSCH, 2000).

Contudo, diversas críticas recaem sobre o suporte gerencial fornecido pelo $\mathrm{ABC}$, tais como as apresentadas por Corbett Neto (1997), Geri e Ronen (2005), que argumentam que este método de custeio não trata adequadamente as restrições existentes nas empresas. Sob o aspecto da restrição, é possível que estas críticas estejam relacionadas com aquelas apresentadas por Goldratt e Cox (1997) no best-seller intitulado "A Meta”. Esta obra trata da Teoria das Restrições (Theory of Constraints - TOC) como uma perspectiva para o suporte à tomada de decisão. Discute-se sobre o fato de as informações de custos não serem o foco da tomada de decisão, e sim, as informações relacionadas a ganhos. Além disso, os autores fazem críticas incisivas quanto aos vários problemas e vieses inerentes às informações de custos - e consequientemente do $\mathrm{ABC}$, que normalmente fazem com que os gestores tomem decisões inadequadas.

Outras críticas, algumas fundamentadas na TOC, também censuram a utilização das informações provenientes do ABC para subsidiar a tomada de decisão. Segundo Roni e Geri (2005): a) a definição dos direcionadores pode recair na subjetividade se não for bem determinada; b) a desconsideração de restrições, tal como a não identificação de gargalos (processos ou atividades com restrição de recursos) pode ser prejudicial às decisões dos gestores; e, c) a pressuposição de que a relação entre o nível de uma atividade e o consumo de recursos é linear.

Ademais, podem-se citar ainda alguns modelos desenvolvidos nas últimas décadas que têm como intuito, dentre outros objetivos, superar as limitações apresentadas pela contabilidade de custos como um todo. Dentre esses modelos, pode-se destacar o modelo de Gestão Econômica (GECON), elaborado, inicialmente, pelo professor brasileiro Armando Catelli no final da década de 1970 e que, segundo Pontes (2003), faz uso de um conjunto de critérios de mensuração bem distinto daquele adotado normalmente pela contabilidade, pois considera que o resultado da empresa deve refletir a agregação de riqueza ocorrida no período. Ainda segundo a autora, o modelo GECON busca medir o valor econômico da empresa e incorpora o conceito de custo de oportunidade, sendo geralmente mais adequado ao subsídio à tomada de decisão gerencial que os métodos de custeio existentes, dentre eles, o ABC. Catelli e Guerreiro (1993, p. 1) afirmam que a fraqueza do ABC (em oposição ao GECON) está relacionada:

fundamentalmente à distribuição de custos fixos aos produtos, ou seja, a utilização de cost drivers ao invés de outros critérios de rateio, não modifica o comportamento intrínseco de parte substancial dos custos das atividades de apoio que é de natureza fixa. Outro aspecto do $\mathrm{ABC}$ diz respeito ao modelo de decisão do usuário da sua informação, ou seja um modelo de decisão ao nosso ver incompleto baseado especificamente no custo. 
Por fim, alguns autores que defendem o ABC como ferramenta gerencial também reconhecem como plausíveis algumas críticas ao referido método de custeio, tais como Kaplan e Anderson (2007a). Segundo estes autores, o ABC tem uma série de limitações, tais como: a morosidade no fornecimento de informações e os altos custos de manutenção. Entretanto, os referidos autores apresentam uma possível solução para parte dessas limitações que pode ser compreendida como um aperfeiçoamento: o TDABC. Esta nova abordagem não abandona totalmente o conceito de $\mathrm{ABC}$; contudo, remodela-o, no intuito de suprir principalmente as limitações da morosidade e do alto custo de manutenção do $\mathrm{ABC}$.

\section{A ABORDAGEM TIME-DRIVEN}

Segundo Bruggeman et al. (2005), o TDABC foi desenvolvido em 1997 por Steven Anderson e implementado em algumas organizações. Em 2001, junto com o professor da Harvard Business School, Robert Kaplan, Anderson aperfeiçoou essa metodologia. Kaplan e Anderson (2007a) destacam-se que essa nova abordagem do ABC já foi implantada em mais de 100 empresas, apresentando bons resultados. Entretanto, ressalta-se o fato de os autores não apresentarem, em seu estudo, maiores detalhes sobre essa afirmação.

De acordo com Kaplan e Anderson (2004), o TDABC visa solucionar as limitações encontradas no ABC, tais como: morosidade, inflexibilidade e onerosidade de implantação e manutenção. Segundo os mesmos autores, essa nova abordagem, além de superar estas limitações, oferece informações úteis e transparentes que refletem as complexidades das operações usualmente realizadas pelas empresas. Ao mesmo tempo, o TDABC é, teoricamente, de rápida implantação e apresenta onerosidade reduzida. Tal metodologia permite a geração de informações mais acuradas para empresas de pequeno, médio e grande porte (KAPLAN; ANDERSON, 2007b).

Conforme Everaert e Bruggeman (2007), o TDABC necessita da estimativa de apenas duas variáveis para sua operacionalização: o custo de fornecimento de recursos a determinada atividade e o tempo requerido para executá-la. Esses autores apresentam seis passos para a implantação do TDABC em uma empresa: 1) identificação dos recursos fornecidos às atividades, segregando-os em grupos; 2) estimativa dos custos de cada recurso; 3 ) mensuração da capacidade prática das atividades; 4) cálculo da unidade de custo de cada recurso - dividese o valor encontrado para cada grupo de recursos pela capacidade prática da atividade; 5) determinação do tempo requerido por cada evento de uma atividade, baseada em diferentes direcionadores de custos; e, 6) multiplicação do custo unitário (obtido no passo 4), pelo tempo requerido por cada objeto de custo (obtido no passo 5).

Quanto aos direcionadores, sob a abordagem do TDABC, eles podem ser classificados como contínuos, discretos ou indicadores (BRUGGEMAN et al., 2005). O peso de um pallet e uma distância em quilômetros podem exemplificar os direcionadores contínuos. Quanto aos direcionadores classificados como discretos, podem ser citados os números de ordens de produção e de verificações de créditos. Por fim, o tipo de clientes e o tipo de encomendas podem ser citados como direcionadores indicadores. Os mesmos autores também ressaltam a importância da inter-relação entre os direcionadores de cada atividade.

Em cada transação executada pela empresa são realizadas diversas atividades, algumas são muito simples, outras, bastante complexas e que exigem diferentes recursos. Kaplan e Anderson (2007a) ressaltam que o TDABC incorpora as variações de tempo demandadas para cada tipo de transação. Isso é possível, devido à utilização das equações de tempo (time equations). Estas equações refletem as diferentes circunstâncias sob as quais uma determinada atividade pode ser desempenhada, devido ao uso de diferentes direcionadores e de suas interações. 
No intuito de esclarecer melhor a elaboração e a utilização das time-equations, apresenta-se um exemplo proposto por Bruggeman et al. (2005). Nesse exemplo, tem-se a atividade processor ordem de pedido, que depende de três direcionadores de tempo: o tipo de cliente (novo ou já existente), o número de linhas de itens por pedido e o tipo de ordem (normal ou urgente). Nesse caso, há dois direcionadores indicadores (tipo de cliente e tipo de ordem) e um discreto (número de linhas de pedido). Quanto aos direcionadores indicadores, esses assumem o valor de "0" (zero) ou "1", sendo que este último só é apresentado quando o cliente for um novo cliente (tipo de ordem) ou a ordem for urgente (tipo de ordem). Com base em estudos e nas variáveis discutidas, poder-se-ia desenvolver a seguinte time-equation:

$$
\text { Tempo_apurado }=X_{1} \times 2+X_{2} \times 15+X_{3} \times 10+3
$$

De acordo com a referida time-equation, a variável $\mathrm{X}_{1}$ representa o direcionador número de linhas da ordem, a variável $\mathrm{X}_{2}$ representa a dicotomia cliente novo/cliente antigo e a variável $\mathrm{X}_{3}$, a dicotomia ordem normal/ordem urgente. Ademais, o valor "3" apresentado na equação se refere ao seu valor independente. Assim, com base na equação desenvolvida, temse que o tempo demandado para o processamento de uma ordem de pedido com 5 linhas para um novo cliente, que solicita uma resposta urgente por parte da empresa é de 38 minutos.

Kaplan e Anderson (2007b) apresentam uma série de vantagens advindas da utilização do TDABC: a) facilidade e rapidez na construção de um modelo acurado; b) integração com os sistemas de gestão integrada (Enterprise Resource Planning - ERPs) e sistemas de relacionamento com clientes; c) direcionadores que refletem as diversas características especiais identificadas em algumas atividades; d) facilidade de utilização em uma base mensal, permitindo o feedback dos gestores rapidamente; e) disponibilidade de informações acuradas sobre a eficiência de processos e a capacidade utilizada de produção; f) boas estimativas sobre o consumo de recursos; g) facilidade de integração com os diversos sistemas existentes nas grandes empresas; h) baixo custo de manutenção; i) disponibilidade de informações que permitem a identificação da origem dos problemas; e, j) utilização em indústrias com grande e complexa variedade de produtos, clientes, canais de distribuição e despesas.

No intuito de elucidar as diferenças entre o ABC e o TDABC, é apresentado a seguir um exemplo proposto por Barret (2005). Por questão de simplicidade, o autor manteve, nas duas abordagens, os mesmos direcionadores para os recursos, a fim de apurar os custos de ambas as formas.

O Departamento $\mathrm{X}$ de uma empresa, entidade hipotética tratada neste exemplo, executa duas atividades: processar requerimentos e cobrar pagamentos atrasados. No mês anterior, o Departamento $\mathrm{X}$ processou 5.000 requerimentos e cobrou 1.000 pagamentos atrasados. Este departamento conta com quatro pessoas, que trabalham em média 7 horas por dia, 20 dias por mês. Os custos diretos do Departamento X (salários, benefícios, dentre outros) totalizam $\$ 16.800,00$. Além disso, $\$ 2.800,00$ (parte dos gastos com o gerente responsável por supervisionar este departamento) e $\$ 4.200,00$ (custos com instalações e tecnologia da informação - custos indiretos) são alocados ao departamento em questão. $\mathrm{O}$ gerente do Departamento $\mathrm{X}$ estima que os tempos de seus funcionários dedicados às atividades desse departamento podem ser assim divididos: 66,67\% dedicados à atividade processar requerimentos e 33,33\% dedicados à atividade cobrar pagamentos atrasados. Os demais custos alocados ao Departamento $\mathrm{X}$ podem ser igualmente divididos entre as atividades (50\% para cada uma delas). A capacidade ociosa do Departamento X é irrisória e, portanto, não precisa ser considerada nos cálculos de custos. 
Com base nos dados acima, os custos são calculados primeiramente pelo ABC (Tabela 1) e, posteriormente, pelo TDABC (Tabela 2). Os dados utilizados para gerar as informações apresentadas na Tabela 2 foram os mesmos utilizados para apurar as informações apresentadas na Tabela 1. Contudo, para que fosse possível mensurar os custos das atividades pela metodologia do TDABC, foi necessária a apresentação dos tempos unitários para executar cada um dos trabalhos. Esses tempos são de 4 minutos para processar um requerimento e 10 minutos para cobrar um pagamento atrasado.

Tabela 1 - Custos apurados pelo ABC

\begin{tabular}{l|r|r}
\hline \multicolumn{1}{c|}{ Atividade } & Processar requerimentos & Cobrar pagamentos atrasados \\
\hline Tempo despendido pelos funcionários (em & 66,67 & 33,33 \\
$\%$ ) & & \\
Alocação de custos diretos (em R\$) & $11.200,00$ & $5.600,00$ \\
Alocação dos custos com supervisão (em R\$) & $1.400,00$ & $1.400,00$ \\
Alocação de custos indiretos (em R\$) & $2.100,00$ & $2.100,00$ \\
Total dos custos (em R\$) - A & $14.700,00$ & $9.100,00$ \\
Número de direcionadores (em unidades) - B & 5.000 & 1.000 \\
Taxa de custo por direcionador (em R $\$$ ) - & 2,94 & 9,10 \\
A/B & & \\
\hline
\end{tabular}

Fonte: adaptado de Barret (2005).

\section{Tabela 2 - Custos apurados pelo TDABC}

\begin{tabular}{l|r|r}
\multicolumn{1}{c|}{ Atividade } & \multicolumn{1}{c}{$\begin{array}{c}\text { Processar } \\
\text { requerimentos }\end{array}$} & $\begin{array}{c}\text { Cobrar pagamentos } \\
\text { atrasados }\end{array}$ \\
\hline Tempo despendido pelos funcionários (em \%) & 66,67 & 33,33 \\
Alocação de custos diretos (em R\$) & $11.200,00$ & $5.600,00$ \\
Alocação dos custos com supervisão (em R\$) & $1.400,00$ & $1.400,00$ \\
Alocação de custos indiretos (em R\$) & $2.100,00$ & $2.100,00$ \\
Total dos custos (em R\$) - A & $14.700,00$ & $9.100,00$ \\
Tempo de estimado (em minutos) - B & $22.400,00$ & $11.200,00$ \\
Custo por minuto (em R\$) - A/B & 0,66 & 0,81 \\
Número de direcionadores (em unidades) - C & 5.000 & 1.000 \\
Tempo para execução da atividade (em minutos) - D & 4,00 & 10,00 \\
Tempo total dispendido (em minutos) - C x D & $20.000,00$ & $10.000,00$ \\
Custo total alocado (em R\$) (C x D) x (A / B) = E & $13.125,00$ & $8.125,00$ \\
Taxa de custos por direcionador (em R\$) & 2,63 & 8,13 \\
Capacidade ociosa & Em R\$ & $2.550,00$ \\
\hline
\end{tabular}

Fonte: adaptado de Barret (2005).

Percebe-se uma informação importante da Tabela 2: a capacidade ociosa relativamente alta apresentada pelo Departamento X. Tal informação havia sido omitida na Tabela 1, elaborada sob a abordagem convencional do ABC. Nela, as informações foram baseadas na estimativa agregada do gerente, que acreditava não existir uma taxa relevante de ociosidade em seu departamento.

\section{METODOLOGIA}

Esta pesquisa, de natureza qualitativa e exploratória, adotou como método de pesquisa o estudo de caso realizado em uma EPE da região metropolitana de Belo Horizonte/MG, que utiliza o modelo tradicional ABC. Conforme Cassel e Symon (1994), a abordagem da pesquisa qualitativa e exploratória se refere à utilização de métodos geralmente associados ao levantamento e a análise de um texto escrito ou falado, ou a uma observação direta de um 
comportamento pessoal. A abordagem utilizada é especialmente relevante para casos nos quais os temas são emergentes e as descrições são ideográficas, visando discutir os assuntos com um maior grau de intensidade. No que tange aos estudos de casos, Yin (2005) afirma que eles se caracterizam pela observação direta, participante ou não, dos acontecimentos contemporâneos, dentro do seu contexto da vida real, principalmente quando os limites entre o contexto prático (real) e o fenômeno (teoria) ainda não são bem definidos.

Para a coleta de dados foram utilizadas diversas técnicas: entrevistas semiestruturadas, análise documental e observação participante. Segundo Hair et al. (2005), as entrevistas semi-estruturadas são cada vez mais utilizadas pelos pesquisadores, pois permitem o surgimento de informações inesperadas e esclarecedoras com um planejamento relativamente aberto, em contraposição à entrevista estruturada (padronizada) ou questionário. Por sua vez, conforme Bardin (2002), a análise documental pode ser realizada a partir de qualquer registro escrito ou em meio magnético usado como fonte de informação. Tedlock (2005) conceitua observação participante como uma técnica de coleta de dados, na qual o pesquisador está fisicamente presente e monitora pessoalmente o que ocorre.

O roteiro de entrevistas enfocava quatro aspectos principais: a) a opinião do funcionário quanto aos direcionadores utilizados para alocar os custos de sua atividade principais aos objetos de custos; b) a opinião do funcionário quanto à utilização de um novo direcionador; c) a possibilidade da segregação das atividades desempenhadas em subatividades, o que permitiria determinar o tempo de cada sub-atividade como padrão e, considerando diversos eventos potenciais, estimar as time equations; e, d) a viabilidade de consecução de informações de tempo de todas as sub-atividades (relação custo-benefício). A análise documental, por sua vez, foi realizada em registros físicos e magnéticos do Departamento de Custos da empresa. Tais registros consistem em planilhas eletrônicas de apuração de custos e relatórios periódicos desenvolvidos pelos funcionários desse departamento. A observação participante foi realizada na fábrica da empresa. Atividades complexas, como usinagem e polimento de aços, foram acompanhadas pelos pesquisadores. Destaca-se que os supervisores de produção esclareceram as dúvidas quanto aos aspectos técnicos dos trabalhos desempenhados no chão-de-fábrica da empresa.

Os dados coletados por meio das entrevistas foram analisados qualitativamente com base na análise de conteúdo. Para Bardin (2002), a análise de conteúdo abrange as iniciativas de explicitação, sistematização e expressão do conteúdo de mensagens. Tal análise objetiva efetuar deduções lógicas e justificadas a respeito da origem das referidas mensagens - o emissor, o contexto, assim como os efeitos que se pretende causar por meio delas. Além disso, foi realizada uma triangulação dos dados coletados pelos diferentes métodos durante a pesquisa. Yin (2005) conceitua triangulação como uma técnica que utiliza diversos dados distintos sobre um determinado fenômeno, no intuito de ratificar os resultados encontrados e aumentar a confiabilidade dos dados coletados.

\section{ESTUDO DE CASO}

\subsection{A Empresa Alfa e uma Análise da Aplicação do $A B C$ Convencional nas Atividades Escolhidas}

A EPE, objeto de estudo deste trabalho, pertence ao setor de ferramentaria, produzindo máquinas-ferramentas para a estampagem de peças utilizadas no setor automotivo. No sentido de resguardar informações sigilosas da EPE, será utilizado o pseudônimo Empresa Alfa. Essa empresa foi selecionada por classificar-se como uma VMC e utilizar o modelo tradicional do $\mathrm{ABC}$ para auxiliar nas decisões gerenciais. 
Destaca-se que a Empresa Alfa implantou o $\mathrm{ABC}$ em nível estratégico. Embora a literatura não seja explícita, sugere-se que o TDABC seja utilizado quando o $\mathrm{ABC}$ está implantado em nível operacional. Por isso, foi utilizado o termo sub-atividades (subdivisão das atividades identificadas na EPE estudada), tal como foi apresentado na seção 5. Elas são a base para o desenvolvimento das time equations, fundamentais para o TDABC.

$\mathrm{Na}$ Empresa Alfa, as horas registradas pelos funcionários são consideradas os direcionadores de custos da maioria das atividades. Quatro atividades, contudo, têm direcionadores distintos: o número de funcionários serve como direcionador para a atividade Gerenciar Pessoal; a atividade Controlar Finanças tem como direcionador o número de notas fiscais apuradas; e as dimensões apuradas das ordens de serviço são os direcionadores de Transportar materiais e Planejar e Controlar a Produção. Cada um destes direcionadores é identificado e mensurado mensalmente.

Para realizar o estudo proposto, foram selecionados quatro funcionários de três atividades distintas: Controlar Finanças, Planejar e Controlar a Produção, e Programar CNC (Computer Numerical Control). Essas atividades foram escolhidas por apresentarem direcionadores distintos. Além disso, foram consideradas as opiniões dos funcionários do Departamento de Custos da empresa (por meio de entrevistas semi-estruturadas). O Quadro 1 apresenta os funcionários entrevistados e suas respectivas atividades. Para manter sigilo dos funcionários, são utilizados pseudônimos, tais como F1 e F2.

\begin{tabular}{|l|l|l|}
\hline \multicolumn{1}{|c|}{ Funcionário } & \multicolumn{1}{c|}{ Atividade } & \multicolumn{1}{c|}{ Direcionador de custos } \\
\hline F1 & Controlar Finanças & Número de notas fiscais apuradas \\
\hline F2 e F3 & Planejar e Controlar a Produção & Dimensões de ordens de serviço \\
\hline F4 & Programar CNC & Tempo apontado pelo funcionário \\
\hline
\end{tabular}

\section{Quadro 1 - Funcionários entrevistados}

Fonte: elaboração própria.

A seguir é descrito a maneira como os custos de cada atividade estudada são alocados às ordens de serviços. Os custos da atividade Controlar Finanças são distribuídos às ordens de serviço por meio do número de notas fiscais de entrada apuradas no período. O fundamento lógico deste direcionador é: quanto maior o número de notas fiscais para cada ordem de serviço, maior o tempo gasto pelos funcionários em cada uma (maior número de itens a serem comprados, negociados com fornecedores e clientes, pagos e contabilizados). Estão envolvidos nesta atividade os departamentos de Compras, Financeiro e Fiscal.

Como exemplo do uso desse direcionador, considere que a Empresa Alfa apurou 10 notas fiscais no período e o custo total da atividade (funcionários, depreciação, licenças de software, dentre outros) foi de $\mathrm{R} \$ 100,00$. O custo por direcionador da atividade foi de $\mathrm{R} \$ 10,00$. Desse modo, ao se mensurar o número de notas fiscais apuradas no período, foram alocados a cada ordem de serviço $\mathrm{R} \$ 10,00$ por nota contabilizada. A Tabela 3 apresenta um exemplo desta alocação de custos a ordens de serviços hipotéticas (A, B e C).

Tabela 3 - Alocação hipotética de custos da atividade Controlar Finanças

\begin{tabular}{c|c|c}
\hline Ordens de serviço & $\begin{array}{c}\text { Número de notas fiscais } \\
\text { apuradas por ordem de serviço }\end{array}$ & $\begin{array}{c}\text { Valor alocado a cada ordem de } \\
\text { serviço }\end{array}$ \\
\hline A & 5 & 50,00 \\
B & 3 & 30,00 \\
C & 2 & 20,00 \\
\hline Total & $\mathbf{1 0}$ & $\mathbf{1 0 0 , 0 0}$ \\
\hline
\end{tabular}

Fonte: elaboração própria. 
Ressalta-se que esse direcionador possui algumas limitações relevantes. O número de notas fiscais não representa, de forma homogênea, o tempo despendido por cada funcionário na atividade (enquanto dez notas fiscais podem ser processadas em uma hora, eventualmente apenas uma nota pode demorar duas horas para ser processada, por exemplo). Ademais, uma nota fiscal pode apresentar materiais e serviços contratados para mais de uma ordem de serviço.

Os custos da atividade Planejar e Controlar a Produção são alocados às ordens de serviço (objetos de custos) por meio das dimensões das ordens de serviço apuradas no período. $\mathrm{O}$ fundamento lógico deste direcionador é: quanto maior o produto fabricado, mais tempo os funcionários da atividade devem dedicar ao mesmo - devido ao maior número de itens, há maior número de fornecedores a serem acompanhados e mais atividades a serem planejadas e coordenadas. Fazem parte desta atividade três funcionários. Para o cálculo deste 3 direcionador, cada produto recebeu uma classificação baseada em , sendo seis categorias possíveis, denominadas scores.

A mensuração é realizada com base no projeto do produto. Assim, se a empresa apurou no período um score total de 10 e o custo total da atividade (funcionários, depreciação, energia elétrica, dentre outros) foi de $\mathrm{R} \$ 100,00$, o custo por direcionador da atividade foi $\mathrm{R} \$ 10,00$. Desse modo, ao se mensurar as dimensões totais das ferramentas no período, seriam alocados $\mathrm{R} \$ 10,00$ a cada ordem de serviço por score que tiver a ordem. A Tabela 4 apresenta um exemplo desta alocação de custos a ordens de serviços hipotéticas (A, B e C).

Tabela 4 - Alocação hipotética de custos da atividade Planejar e Controlar a Produção

\begin{tabular}{c|cc}
\hline $\begin{array}{c}\text { Ordens de } \\
\text { serviço }\end{array}$ & Score das ordens de serviço & $\begin{array}{c}\text { Valor alocado a cada ordem de serviço } \\
\text { (em R } \mathbf{\text { ) }}\end{array}$ \\
\hline A & 5 & 50,00 \\
B & 3 & 30,00 \\
C & 2 & 20,00 \\
\hline Total & $\mathbf{1 0}$ & $\mathbf{1 0 0 , 0 0}$ \\
\hline
\end{tabular}

Fonte: elaboração própria.

Destaca-se que este direcionador também possui algumas limitações. A classificação da ordem de serviço não representa homogeneamente o tempo despendido por funcionário na atividade. Isto porque uma ordem de serviço de pequenas dimensões pode eventualmente demandar um número maior de programações de máquinas do que uma de maior porte, por exemplo.

Por fim, os custos da atividade Programar CNC são alocados às ordens de serviço por meio do número de horas apuradas no período. O fundamento lógico deste direcionador é: quanto maior o número de horas trabalhadas em cada ordem de serviço, maior o tempo dedicado pelos funcionários a cada uma. Neste caso, o custo dos funcionários é considerado um custo direto.

Considerando que a empresa apurou 10 horas de trabalho no período e o custo total da atividade (funcionários, depreciação, licenças de software, dentre outros) foi de $\mathrm{R} \$ 100,00$, o custo por direcionador da atividade foi de $\mathrm{R} \$ 10,00$. Desse modo, ao mensurar o número de horas por período, os custos alocados a cada ordem de serviço foram de $\mathrm{R} \$ 10,00$ por hora de trabalho. A Tabela 5 apresenta um exemplo deste custeio de ordens de serviços hipotéticas (A, B e C). 
Tabela 5 - Alocação hipotética de custos da atividade Programar CNC

\begin{tabular}{c|c|c}
\hline $\begin{array}{c}\text { Ordens de } \\
\text { serviço }\end{array}$ & $\begin{array}{c}\text { Número de horas dedicadas a cada } \\
\text { ordem de serviço }\end{array}$ & $\begin{array}{c}\text { Valor alocado a cada ordem de serviço } \\
\text { (em R\$) }\end{array}$ \\
\hline A & 2 & 20,00 \\
B & 2 & 20,00 \\
C & 6 & 60,00 \\
Total & $\mathbf{1 0}$ & $\mathbf{1 0 0 , 0 0}$ \\
\hline
\end{tabular}

Fonte: elaboração própria.

Dos três direcionadores apresentados e analisados nesta pesquisa, verifica-se que o direcionador número de horas apuradas nos períodos é o mais adequado para a Empresa Alfa. Os custos são, neste caso, considerados diretos, o que atenua vieses em sua alocação. A principal limitação deste direcionador é a dependência das informações repassadas pelos funcionários da empresa. Essas informações podem ser manipuladas pelos funcionários, e, por esse motivo, levar a distorções no processo de mensuração dos custos. Com exceção do direcionador da última atividade apresentada, os demais apresentam vieses que podem prejudicar a qualidade da informação de custos para a gestão e tomada de decisão, tanto em nível operacional quanto estratégico. Contudo, ao realizar uma análise do custo-benefício, os erros decorrentes das abordagens parecem ser superados pela qualidade das informações disponibilizadas.

\subsection{Percepção dos Gestores Sobre a Aplicação do TDABC nas Atividades Selecionadas}

A funcionária responsável pela atividade Controlar Finanças (F1) concorda com o uso do direcionador de custo utilizado no atual modelo $A B C$ da sua atividade e não acredita na possibilidade do uso de algum outro. A referida funcionária também afirma que é possível a segregação de sua atividade em outras sub-atividades padronizadas. Segundo ela, poderiam ser estimados tempos para a execução da maioria de suas atividades diárias. Contudo, algumas outras atividades realizadas pela funcionária a impedem de estimar com exatidão os seus tempos de trabalho.

Sobre a estimativa do tempo utilizado para as sub-atividades, a funcionária comenta, exemplificando, que uma sub-atividade cujo tempo poderia ser previsto é a de elaboração de ordens de compra. O tempo gasto, neste caso, depende do número de itens listados no projeto da ferramenta. Contudo, uma dificuldade para medir o tempo é o fato de, muitas vezes ao dia, a necessidade de parar para atender telefonemas e resolver problemas cuja estimativa de tempo é praticamente inexeqüível. Assim, tem-se que estas sub-atividades paralelas, além de não serem passíveis de padronização e previsão, freqüentemente influenciam as subatividades mais rotineiras. Durante a elaboração de uma ordem de compra, por exemplo, a funcionária pode parar diversas vezes e dedicar um tempo razoável àquelas atividades, cujo tempo para a execução é normalmente imprevisível.

Por sua vez, os funcionários entrevistados responsáveis pela atividade Planejar e Controlar a Produção (F2 e F3) discordam do uso do direcionador de custo utilizado no atual modelo ABC. Segundo eles, diversas peculiaridades de cada ordem de serviço tornam o direcionador baseado em dimensões, em grande parte, incoerente. Conforme esses funcionários, algumas ferramentas pequenas são mais complexas e demandam mais tempo de produção do que outras maiores. Ressalta-se que tal fato é, em parte, considerado no atual processo de alocação de custos às ordens de serviço, pois, uma vez que a ferramenta complexa permanece mais tempo na fábrica, os custos com a atividade Planejar e Controlar a Produção serão alocados à ela por um tempo maior, considerando seus custos de complexidade. 
Uma proposta apresentada pelo funcionário F3 foi a classificação das ferramentas em duas categorias: as fabricadas com base em fundidos e as fabricadas com base em aço laminado. A justificativa do funcionário foi que as ferramentas baseadas em fundidos demandam poucos esforços antes da produção na fábrica, enquanto as com base em aço laminado demandam mais esforços neste período. Por outro lado, na fase de produção da ferramenta, a situação se inverte, uma vez que os laminados envolvem um conjunto menor de atividades, enquanto os fundidos demandam um grande conjunto de atividades.

Quanto à previsibilidade e à estimação de tempo de suas sub-atividades, os funcionários entrevistados julgaram ser uma tarefa impossível na empresa estudada. Segundo eles, suas atividades demandam tempos bastante discrepantes e imprevisíveis, o que impede a realização de estimativas confiáveis de tempos para as mesmas. Um caso citado pelos funcionários para exemplificar a afirmativa apresentada é a supervisão do desenvolvimento de ferramentas terceirizadas. Muitas vezes, o funcionário responsável por acompanhar as ferramentas elaboradas em outras empresas consegue ir às fábricas de diversos fornecedores (empresas terceirizadas) em apenas um dia. Contudo, além do número de empresas visitadas ser bastante variável, dependendo dos diversos fatores que as influenciam, muitas dessas visitas, que inicialmente parecem simples, tornam-se bastante complexas. Algumas vezes, o funcionário responsável por supervisionar os produtos terceirizados consegue visitar apenas uma empresa durante todo o dia.

Por fim, o funcionário entrevistado da atividade Programar $\mathrm{CNC}(\mathrm{F} 4)$ não concorda plenamente com o uso do direcionador de custo utilizado no atual modelo ABC para alocar os custos das suas atividades às ordens de serviço. Contudo, suas críticas são pontuais e fogem ao escopo do presente trabalho. Quanto à possibilidade da estimação dos tempos de suas subatividades, o funcionário afirma ser inviável realizar tal tarefa. Segundo ele, muitas de suas sub-atividades são realizadas sob circunstâncias únicas, o que impede uma previsão do tempo de execução da mesma. Tal fato também parece comprovar a complexidade do trabalho em empresas de produção por encomenda.

Um exemplo apresentado por F4 para sustentar sua afirmativa é a sub-atividade de modelagem. Tal trabalho consiste em transformar um desenho (projeto) de duas dimensões para outro, em três dimensões, de forma que uma máquina CNC possa usiná-lo. O tempo de modelagem gasto em cada produto varia de acordo com uma série de fatores, tais como a complexidade e a dimensão da peça a ser estampada. Além disso, o entrevistado afirmou que a modelagem de cada ferramenta varia de acordo com o trabalho executado pelo funcionário, ou seja, ela pode ser realizada de diversas maneiras, com tempos distintos. Assim, a modelagem pode alcançar o mesmo resultado (eficácia); todavia, a eficiência é variável. A síntese dos resultados obtidos nas entrevistas semi-estruturadas realizadas com os funcionários F1, F2, F3 e F4 são apresentados no Quadro 2.

\begin{tabular}{|c|c|c|c|c|}
\hline \multicolumn{2}{|c|}{ Funcionários } & \multirow{2}{*}{\begin{tabular}{c|} 
F1 \\
Controlar \\
Finanças \\
\end{tabular}} & \multirow{2}{*}{$\begin{array}{c}\text { F2 e F3 } \\
\begin{array}{c}\text { Planejar e Controlar } \\
\text { a Produção }\end{array} \\
\end{array}$} & \multirow{2}{*}{$\begin{array}{c}\text { F4 } \\
\text { Programar CNC }\end{array}$} \\
\hline & Atividades & & & \\
\hline \multirow{4}{*}{ 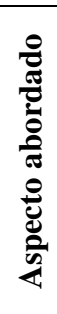 } & $\begin{array}{llll}\begin{array}{l}\text { Opinião } \\
\text { utilizados }\end{array} & \text { quanto } & \text { aos } & \text { direcionadores } \\
\end{array}$ & Adequados & Inadequados & $\begin{array}{l}\text { Adequados (com } \\
\text { restrições) }\end{array}$ \\
\hline & $\begin{array}{l}\begin{array}{l}\text { Possibilidade de utilização } \\
\text { direcionadores }\end{array}\end{array}$ & Não é viável & É viável & Não é viável \\
\hline & $\begin{array}{l}\text { Possibilidade de segregação das atividades } \\
\text { em sub-atividades }\end{array}$ & É viável & Não é viável & Não é viável \\
\hline & $\begin{array}{l}\text { Viabilidade de consecução de informações } \\
\text { de tempos para as sub-atividades }\end{array}$ & É viável & Não se aplica & Não se aplica \\
\hline
\end{tabular}

Quadro 2 - Síntese dos resultados obtidos com base nos roteiros de entrevistas Fonte: elaboração própria. 
Os funcionários do Departamento de Custos da empresa concordam com a opinião dos profissionais entrevistados: não parece viável uma estimativa dos tempos das sub-atividades executadas. Isso se deve principalmente ao fato de o ambiente de uma EPE, tal como a Empresa Alfa, de modo geral, demandar uma grande flexibilidade por parte dos funcionários e das atividades, sendo que alguns problemas a serem resolvidos são únicos e não são passíveis de previsão.

A observação participante das atividades realizada na fábrica corrobora com o apresentado pelos funcionários F2, F3 e F4. A maioria das atividades executadas na empresa é realizada sob circunstâncias únicas, devido ao ambiente instável e flexível de uma EPE. Como já apresentado, os produtos são, normalmente, customizados e as atividades, embora já, em grande parte, pré-definidas, têm que se adaptar ao contexto sob o qual é executada. Pode-se exemplificar tal fato a partir da análise da execução de uma atividade denominada Trabalhar Manualmente a Ferramenta (Bancada). Esta atividade é desempenhada por funcionários denominados ferramenteiros, que devem realizar diversos trabalhos manuais nos aços que compõem a ferramenta. Dentre estes trabalhos, está o de realizar o acabamento na ferramenta por meio de uma pedra-abrasiva. Contudo, tal trabalho depende essencialmente de uma série de variáveis, tais como: dimensões do aço, tipo de aço utilizado, experiência do funcionário, tipo de pedra abrasiva utilizada, dentre outros. Ademais, o supervisor do setor pode decidir realizar o acabamento com o uso de lixadeiras, devido a algumas peculiaridades do trabalho. Assim, torna-se perceptível como a heterogeneidade e o ambiente flexível das EPEs impedem, de forma demasiada, a previsibilidade do tempo necessário.

Por fim, a análise documental, realizada em registros magnéticos e físicos da empresa, também demonstrou a complexidade das operações da empresa e a inviabilidade de estabelecer previsões ambiente operacional da EPE. Todos os controles do Departamento de Custos, por exemplo, têm um escopo bastante extenso, para permitir a inclusão de uma série de operações não planejadas que podem ocorrer (e que normalmente ocorrem). As planilhas eletrônicas de custos utilizadas, por exemplo, possuem diversas contas sem denominação específica (intituladas Contas de Contingência) para registrar transações imprevisíveis e/ou eventuais. Além disso, diversos procedimentos entre os departamentos da empresa são freqüentemente discutidos e revistos, devido à diversidade de fatos imprevistos que normalmente ocorrem durante a execução das atividades diárias.

Desse modo, os resultados encontrados demonstram que o ambiente flexível e de pouca previsibilidade da Empresa Alfa, dificulta, a priori, uma metodologia de cálculo de tempo das atividades, notadamente as relacionadas ao ambiente produtivo. Tal fato impede o desenvolvimento das time equations, que são os fundamentos do TDABC. Destaca-se que, essa impossibilidade de se desenvolver as time equations impede uma análise comparativa entre as vantagens do TDABC apresentadas por Kaplan e Anderson (2007b) em relação às apresentadas pelo modelo atual do $\mathrm{ABC}$ utilizado pela empresa.

\section{CONCLUSÕES}

$\mathrm{O} A \mathrm{ABC}$ foi desenvolvido com o intuito de fornecer informações para melhorar o apoio à tomada de decisão, superando as limitações existentes nos demais métodos de custeio existentes utilizados no ambiente empresarial. Contudo, verifica-se que o ABC também apresenta uma série de limitações, apesar de os benefícios auferidos com sua aplicação serem significativos.

Nesse sentido, uma nova abordagem do ABC, o TDABC, foi desenvolvida recentemente. Essa abordagem inovadora tenta superar ou, pelo menos, atenuar as limitações apresentadas pelo modelo convencional do ABC. A abordagem TDABC explora, de forma 
diferenciada, alguns conceitos já existentes no $\mathrm{ABC}$, assim como apresenta novos conceitos e técnicas.

Este trabalho apresentou os resultados de uma pesquisa que objetivou estudar a percepção dos gestores, de atividades selecionadas em uma EPE localizada na região metropolitana de Belo Horizonte/MG, sobre a aplicabilidade do TDABC Essa empresa já utiliza o método $\mathrm{ABC}$ em sua forma tradicional. Verificou-se que, principalmente em decorrência da grande instabilidade e imprevisibilidade do ambiente de produção por encomenda, a aplicabilidade do TDABC parece ser consideravelmente limitada. Desse modo, considerando as limitações dessa pesquisa, pode-se concluir que as técnicas implantadas pela abordagem TDABC não permitem a sua plena aplicação na EPE estudada.

A partir dos resultados obtidos nesta pesquisa pode-se concluir que a identificação e a definição das time equations é a principal dificuldade para a aplicação do TDABC na EPE estudada Esta dificuldade reside no fato de que as atividades desse tipo de empresa apresentarem grande imprevisibilidade, tanto com relação ao tempo de execução quanto à intensidade do consumo de recursos.

Tendo em vista os resultados apresentados neste trabalho, destacam-se algumas limitações da pesquisa. Primeiramente, os resultados não podem ser generalizados indiscriminadamente, uma vez que se tratou de um estudo de caso único, notadamente em uma EPE classificada como VMC e com o processo de lote. Ademais, a EPE estudada é uma ferramentaria, cujo setor de atuação é bastante peculiar. Ressalta-se também a escassez de pesquisas sobre o TDABC, considerando o recente desenvolvimento dessa ferramenta gerencial. Estudos futuros poderiam explorar essas limitações, analisando a aplicabilidade do TDABC em outras EPEs (tais como as RBCs e em outros processos produtivos) de outros setores.

\section{REFERÊNCIAS}

BASTOS, J. S. Y. Inteligência competitiva: a necessidade de uma prática ética. In: KM Brasil, 2003, São Paulo. Anais... Disponível em: <http://www.netic.com.br/docs/publicacoes /pub0001.pdf >. Acesso em: 16 jun. 2008.

ATKINSON, A. A. et al. Contabilidade gerencial. 2. ed. São Paulo: Atlas, 2008.

BARDIN, L. Análise de conteúdo. Lisboa: Edições 70, 2002.

BARRET, R. Time-Driven costing: the bottom line on the new ABC. Business Performance Management Magazine, March, 2005.

BOISVERT, H. Contabilidade por atividades: práticas avançadas. São Paulo: Atlas, 1999.

BRANDAO, H.P.; GUIMARÃES, T.A. Gestão de competências e gestão de desempenho: tecnologias distintas ou instrumentos de um mesmo construto? In: EnANPAD, 12., Foz do Iguaçu, 1999. Anais... Rio de Janeiro: ANPAD, 1999. CD-ROM.

BRIMSON, J. A. Contabilidade por atividades: uma abordagem de custeio baseado em atividades. São Paulo: Atlas, 1996.

BRUGGEMAN, W.; ANDERSON, S. R.; LEVANT, Y. Modeling logistics costs using TimeDriven ABC: a case in a distribution company. Working Papers of Faculty of Economics and Business Administration, Ghent University, Belgium 05/332, Ghent University, Faculty of Economics and Business Administration, 2005. 
CAMPOS, R. L. O capital intelectual e o processo de estimação de custos e formação de preços em empresas de produção por encomenda. 2003. 152 f. Dissertação (Mestrado em Administração) - Universidade Federal de Minas Gerais, Belo Horizonte, 2003.

CASSEL, C.; SYMON, G. Qualitative research in work contexts. In: CASSEL, C.; SYMON, G. Qualitative methods in organizational research: a practical guide. California: Sage Publications Inc., 1994.

CATELLI, A.; GUERREIRO, R. MENSURAÇÃO DE ATIVIDADES: COMPARANDO ABC” x 'GECON”. Caderno de Estudos FIPECAFI, n. 8, abr., 1993.

COKINS, G. Learning to love ABC. Journal of Accountancy, Aug, p. 37-39, 1999.

COKINS, G.; HICKS, D. Where does the ABC fit amongst the clutter of managerial accounting? Cost Management. v. 21, n. 2, p. 21-28, mar./abr. 2007.

CORBETT NETO, T. Contabilidade de ganhos. São Paulo: Nobel, 1997.

CORRÊA, H. L.; CORRÊA, C. A. Administração de produção e operações - manufatura e serviços: uma abordagem estratégica. 2. ed. São Paulo: Atlas, 2007.

EVERAERT, P. BRUGGEMAN, W. Time-Driven Activity-Based Costing: exploring the underlying model. Cost Management, v. 21, n. 2, p. 16-20, Mar./Apr. 2007.

GERI, N.; RONEN, B. Relevance lost: the rise and fall of activity-based costing. Human Systems Management, v. 24, n. 2, p. 133-144, 2005.

GOLDRATT, E. M.; COX, J. A meta: um processo de aprimoramento contínuo. 39. ed. São Paulo: Educator, 1997.

GRUNOW et al. Custeio e gestão baseado em atividade: o caso Sadia S.A. In: CONGRESSO BRASILEIRO DE CUSTOS, 12., Itapema/SC, 2005. Anais... São Leopoldo, ABC, 2005. CD-ROM.

HAIR, Jr., J. F. et al. Fundamentos de pesquisa em administração. Porto Alegre: Bookman, 2005.

HANSEN, D. R.; MOWEN, M. M. Cost management: accounting and control. 5. ed. Mason Ohio: Thomson/South-Western, 2006.

HENDRY, L. C. Applying world class manufacturing to make-to-order companies: problems and solutions. International Journal of Operations \& Production Management, v. 18, n. 11, p. 1086-1100, Nov. 1998. doi:10.1108/01443579810231679

HIRSCH, M. L. Advanced management accounting. 2. ed. London: Thomson Learning, 2000 .

HUNGARATO, A.; SANT'ANNA, D. Activity Based Costing (ABC) para empresas de distribuição de energia elétrica no Brasil: uma proposta de aplicação. In: CONGRESSO BRASILEIRO DE CUSTOS, 12., Itapema/SC, 2005. Anais... São Leopoldo: ABC, 2005. CD-ROM.

KAPLAN, R. S.; ANDERSON, S. R. Time-Driven Activity-Based Costing. Harvard Business Review, v. 82, n. 11, p. 131-138, Nov. 2004

KAPLAN, R. S.; ANDERSON, S. R. The innovation of Time-Driven Activity-Based Costing. Cost Management, v. 21, n. 2, Mar./Apr., 2007a. 
KAPLAN, R. S.; ANDERSON, S. R. The speed-reading organization. Business Finance, Jun., 2007b.

KAPLAN, R. S.; COOPER, R. Custo e desempenho: administre seus custos para ser mais competitivo. São Paulo: Futura, 1998.

LATSHAW, C. A.; CORTESE-DANILE, T. M. Activity-Based Costing: usage and pitfalls. Review of Business, p. 30-32, Winter 2002.

MEGLIORINI, E. Análise crítica dos conceitos de mensuração utilizados pro empresas brasileiras produtoras de bens de produção por encomenda. 2003. $216 \mathrm{f}$. Tese (Doutorado em Contabilidade e Controladoria) - Universidade de São Paulo, São Paulo, 2003.

PALOMINO, R. Um modelo para o planejamento e a programação da produção em ambientes job shop baseado em redes de Petri. Tese (Doutorado) - Universidade Federal de Santa Catarina. 2001.

PONTE, V. M. R. Modelo de apuração de resultado de redes de varejo sob o enfoque da gestão econômica - GECON. Revista Contabilidade \& Finanças - USP, São Paulo, n. 33, p. 65 - 77, set./dez., 2003

SOUZA, A. A., et al. Análise de sistemas de informações utilizados como suporte para os processos de estimação de custos e formação de preços. In: CONGRESSO BRASILEIRO DE CUSTOS, 12., Itapema/SC, 2005. Anais... São Leopoldo: ABC, 2005. CD-ROM.

STEVENSON, M. A. Review of production planning and control: the applicability of key concepts in the make-to-order industry. International Journal of Production Research. v. 43, n. 5, p. 869-898, Mar. 2005. doi:10.1080/0020754042000298520

TEDLOCK, B. The observation of participation and the emergence of public ethnography. In: DENZIN, N. K.; LINCOLN, Y. S. The sage handbook of qualitative research. 3. ed. California: Sage Publications Inc., 2005.

YEH, C. H. A customer-focused planning approach to make-to-order production. Industrial $\begin{array}{llllllll}\text { Management \& Data Systems, } & \text { v. } 100, & \text { n. } & 4, & \text { p. } & 180-187, & \end{array}$ doi:10.1108/02635570010328693

YIN, R. K. Estudo de caso: planejamento e métodos. 3. ed. Porto Alegre: Bookman, 2005. 